\title{
Recent Progress in Organic Solar Cells based on Small Molecules
}

\author{
Moritz K. Riede ${ }^{a}$, Rico Schueppel ${ }^{a}$, Kerstin Schulze ${ }^{a}$, David Wynands ${ }^{a}$, Ronny Timmreck ${ }^{a}$, \\ Christian Uhrich ${ }^{b}$, Annette Petrich $^{a}$, Martin Pfeiffer ${ }^{b}$, Eduard Brier ${ }^{c}$, Egon Reinold ${ }^{c}$, Peter \\ Baeuerle $^{c}$, Karl Leo ${ }^{a}$ \\ ${ }^{a}$ Institut für Angewandte Photophysik, Technische Universität Dresden, 01069 Dresden, \\ Germany; \\ ${ }^{b}$ Heliatek GmbH, Liebigstr. 26, 01187 Dresden, Germany; \\ ${ }^{c}$ Institut für Organische Chemie II und Neue Materialien, Universitt Ulm, 89069 Ulm, \\ Germany
}

\begin{abstract}
We report on a series of organic solar cells based on heterojunctions of oligothiophene derivatives with varying chain length and C60 fullerenes. Devices are based on either p-i-n or p-i-i structure. In the first the intrinsic photovoltaic active layer is sandwiched between a p-type and n-type doped organic wide-gap layer for hole and electron transport respectively. In the latter the electron transport layer is replaced by a thin layer of wide-gap material as exciton blocker. Through optimization of transport and absorber layers we are able to reach in devices with single heterojunctions an open circuit voltage $V_{\text {oc }}$ of about $1 \mathrm{~V}$, a short circuit current density $J_{\mathrm{sc}}$ of about $5.6 \mathrm{~mA} / \mathrm{cm}^{2}$ and a fill factor $F F$ above $50 \%$ under an AM1.5 illumination with $1000 \mathrm{~W} / \mathrm{m}^{2}$. However, still only a small part of the available solar spectrum is used.

Thus, based on these materials stacked solar cells have been made to further improve the light absorption. The thickness of each layer is optimized using optical simulations to match the currents delivered by each of the solar cells in the stack. Through the incorporation of a very efficient recombination zone between the stacked solar cells the resulting $V_{\text {oc }}$ nearly reaches the sum of the $V_{\text {oc }}$ of the two serially connected solar cells.
\end{abstract}

Keywords: organic solar cells, oligothiophenes, doped transport layer, tandem solar cells

\section{INTRODUCTION}

Organic solar cells have attracted more and more interest in recent years. The prospects of a low-cost production of flexible solar cells on the one hand and the intriguing basic physical properties of the used organic materials on the other hand offer many different starting points for research. First organic solar cells have in the meantime reached efficiencies and lifetimes being close to the ones required for entering the market. ${ }^{1,2}$ Consequently companies have just started production ${ }^{3}$ or are preparing to make them commercially available soon ${ }^{4-6}$ to offer an inexpensive renewable energy source.

Organic solar cells are currently grouped into two main classes according to their underlying working principle: dye sensitized solar cells (DSSC) ${ }^{7}$ and heterojunction solar cells (HJC) ${ }^{8,9}$ Within the group of HJCs one typically differentiates further depending on the production process: solution processing or evaporation of the organic materials. Standard materials for solution processing are currently polythiophenes in combination with fullerene derivatives. The most commonly used materials for photovoltaic heterojunctions based on small molecules are metal phthalocyanines as electron donor and C60 as acceptor. This article focusses on solar cells made with the latter production process.

In this paper we report on a series of organic solar cells based on heterojunctions of recently developed oligothiophene derivatives with varying backbone length and C60 fullerenes. The series of oligothiophenes offer

Further author information: (Send correspondence to M.K.R.) M.K.R.: E-mail: moritz.riede@iapp.de, Telephone: 4935146336043

K.L.: E-mail: karl.leo@iapp.de

Photonics for Solar Energy Systems II, edited by Andreas Gombert

Proc. of SPIE Vol. 7002, 70020G, (2008) - 0277-786X/08/\$18 - doi: 10.1117/12.782232

Proc. of SPIE Vol. 7002 70020G-1 
a)

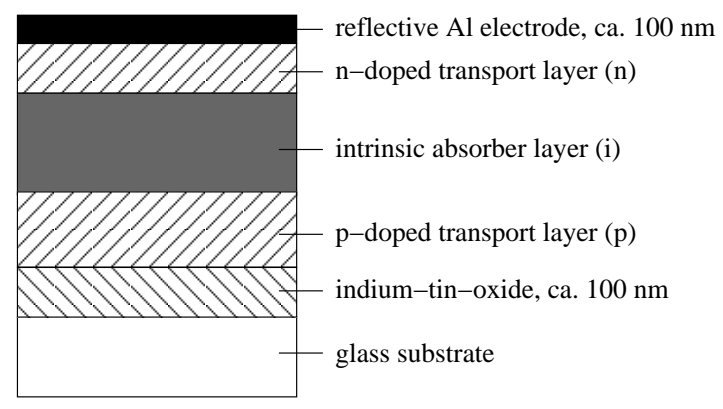

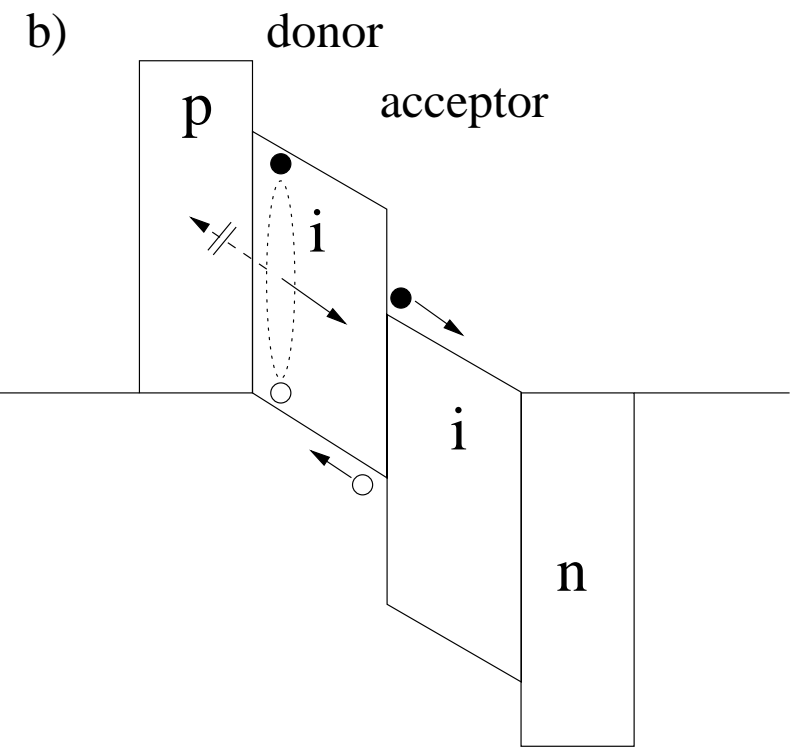

Figure 1. A schematics of the ideal p-i-n structure (a) and corresponding energy diagram (b) for organic solar cells. The photovoltaic active instrinsic layer (i) is sandwiched between p-doped and n-doped wide gap transport materials. It can be either a blend of donor and acceptor as bulk heterojunction or a two layer system as shown in (b) as planar heterojunction.

both an excellent access for the investigation of the basic physical processes in organic solar cells ${ }^{10}$ and the production of efficient solar cells. ${ }^{11}$

\section{EXPERIMENTAL}

The devices are based on either $\mathrm{p}-\mathrm{i}-\mathrm{n}$ or $\mathrm{p}-\mathrm{i}-\mathrm{i}$ structure ${ }^{12}$ and a schematic stack structure and energy diagram of a p-i-n solar cell is shown in figure 1. The intrinsic photovoltaic active layer is sandwiched between transparent transport layers which are doped with molecular dopants as p- and n-type. To simplify the device structure, it is possible to replace the n-doped layer with a thin wide-gap exciton blocker.

The p-i-n structure has several electrical and optical advantages. Due to the doping of the transport layers, typically the contact between transport layer and electrode is ohmic. ${ }^{13}$ The transport layer further can act as semipermeable mebrane, i.e. it only permits the transport of one charge carrier species and thus blocks opposite charge carriers and reflect excitons. Doped transport layers can reach conductivities above $10^{-6} \mathrm{~S} / \mathrm{cm}$ high enough such that ohmic losses over $100 \mathrm{~nm}$ are negligible. ${ }^{13}$ If the transport layers furthermore do not significantly absorb in the sun spectrum, variations in their thickness can be used for optical optimisation of the solar cell stack. ${ }^{14}$ The photovoltaic absorber can subsequently be optimally positioned in the stack with respect to the absorption in the optical interference pattern forming due to the reflecting back contact. In the p-i-i structure the possibilities to tune the position of the optical interference pattern are reduced, because only thin layer can be used between absorber and electron contact. Yet it is often done to simplify the device structure. The general advantages of these device structures are explained in more detail in Ref. ${ }^{12}$

The chemical structures of the molecules used in this paper are shown in figure 2. The photovoltaic active layer consists of a recently developed class of oligothiophenes and the well known fullerene C60 (Kurtschatov Institute, Moscow). The promising oligothiophenes $\alpha, \alpha^{\prime}$-bis-(2,2-dicyanovinyl)-quinquethiophene (DVC5T) with butyl side chains and $\alpha, \alpha^{\prime}$-bis-(2,2-dicyanovinyl)-sexithiophene (DCV6T) with ethyl side chains, were used as electron donor.

N,N'-diphenyl-N,N'-bis(4'-(N,N-bis(naphth-1-yl)-amino)-biphenyl-4-yl)-benzidine (Di-NPB), 4,4'-bis-(N,N-diphenylamino)-quaterphenyl (4P-TPD), 4,4',4 “-tris(1-naphthylphenylamino)-triphenylamine (TNATA) and N,N'diphenyl-N,N'-bis(1-naphthyl)-1-1'biphenyl-4,4" diamine (NPD) were used as hole transport material (HTM, all 


$$
\text { DCV5T }
$$
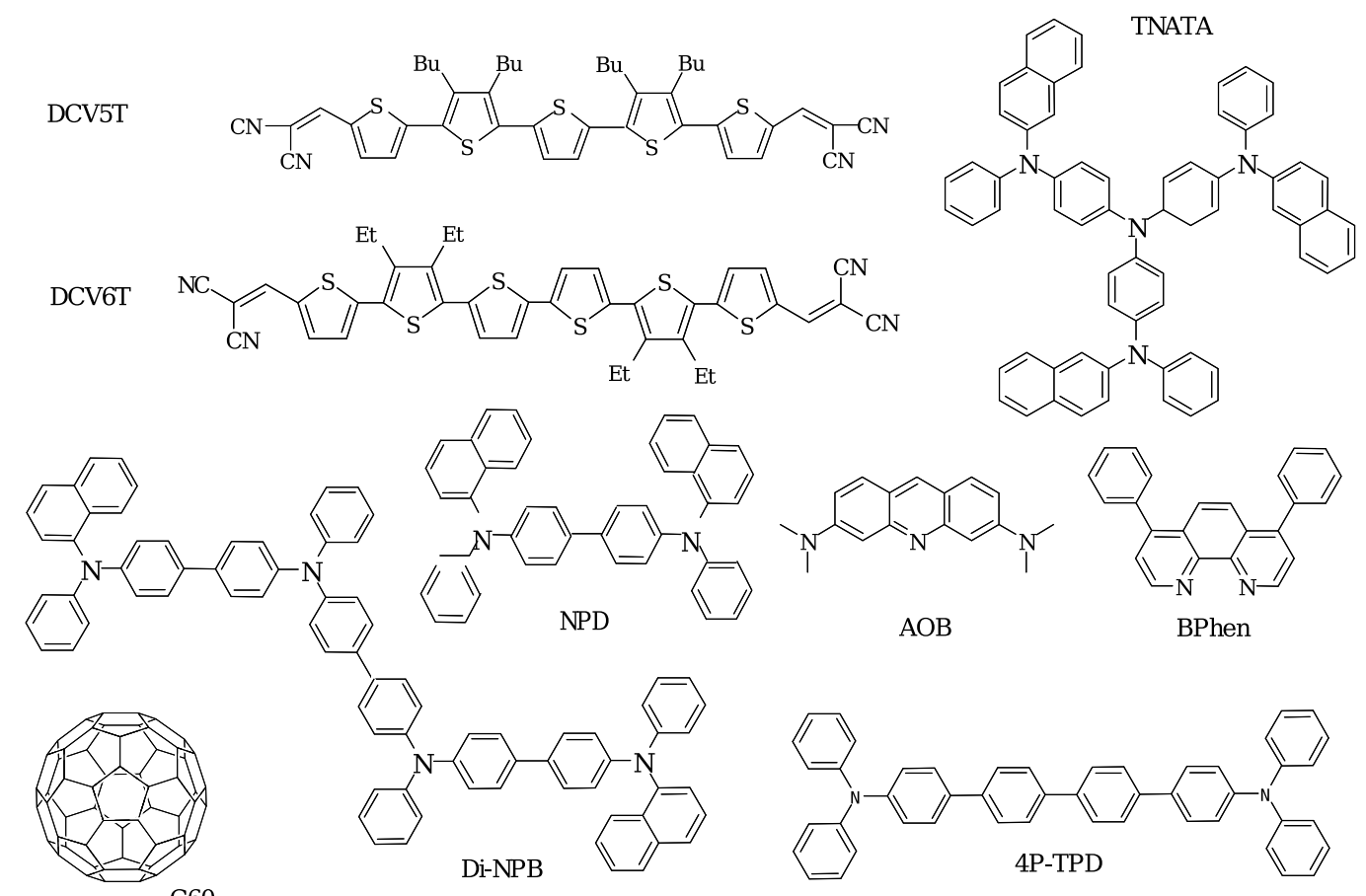

C60

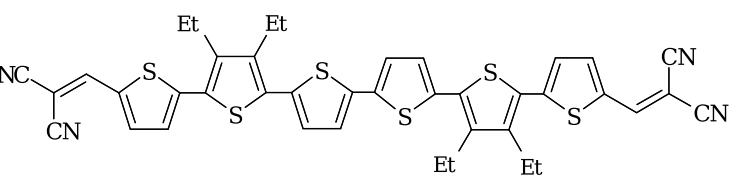

Figure 2. The chemical structure of the used materials. The absorber materials were $\alpha, \alpha^{\prime}$-bis- $(2,2-$ dicyanovinyl)-quinquethiophene (DVC5T) with butyl side chain, $\alpha, \alpha^{\prime}$-bis-(2,2-dicyanovinyl)-sexithiophene (DCV6T) with ethyl side chains and the fullerene C60. N,N'-diphenyl-N,N'-bis(4'-(N,N-bis(naphth-1-yl)-amino)-biphenyl-4yl)-benzidine (Di-NPB), 4,4'-bis-(N,N-diphenylamino)-quaterphenyl (4P-TPD), N,N'-diphenyl-N,N'-bis(1-naphthyl)-11'biphenyl-4,4" diamine (NPD) and 4,4',4"-tris(1-naphthylphenylamino)-triphenylamine (TNATA) were used as hole transport materials. 2,3,5,6-tetrafluoro-7,7,8,8-tetracyanoquinodimethan (F4-TCNQ) and acridine orange base (AOB) are often used as p-type and n-type dopant. 4,7-diphenyl-1,10-phenanthroline (BPhen) is used as exciton blocker.

by Sensient, Wolfen). For the n-side, either C60 was n-doped as eletron transport material or a thin layer of 4,7-diphenyl-1,10-phenanthroline (BPhen) was deposited as exciton blocker.

The p-dopants NDP-2 and NDP-9 from Novaled AG, Dresden were used due to practical reasons instead of the nearly equivalent 2,3,5,6-tetrafluoro-7,7,8,8-tetracyanoquinodimethan (F4-TCNQ). C60 was doped using acridine orange base (AOB).

Due to their favourable values for highest occupied molecular orbital (HOMO) and lowest unoccupied molecular orbital (LUMO) the oligothiophenes are suitable absorber materials in combination with C60. ${ }^{15}$ The HOMO of DVC5T was determined from UPS measurements as - $(5.6 \pm 0.1) \mathrm{eV}$ and the LUMO from CV measurement to be $-3.58 \mathrm{eV}$. For DCV6T we estimate a $\mathrm{HOMO}$ of $-5.5 \mathrm{eV}$ and determined the LUMO with CV to $-3.44 \mathrm{eV}$.

\subsection{Device Production and Characterisation}

Indium-Tin-Oxide (ITO)-coated glass (Thin Film Devices Inc. $\leq 30 \Omega / \square$ ), which had been structured by wet chemical process steps, was used as substrate. Substrates were cleaned in a series of organic solvents. Subsequently they were transfered into a $\mathrm{N}_{2}$-filled glovebox $\left(\mathrm{H}_{2} \mathrm{O} \leq 2 \mathrm{ppm}\right.$ and $\left.\mathrm{O}_{2} \leq 1 \mathrm{ppm}\right)$ and from there into the UHV system in which all further processing steps were carried out. The organic layers and the metal cathode were deposited by thermal evaporation ( $p_{\text {base }} \leq 10^{-6} \mathrm{mbar}$ ) with rates of $0.1-0.4$ and $10-15 \AA / \mathrm{s}$ respectively without breaking the vacuum. Doped transport layers were obtained through co-evaporation of the materials. Deposition rates were controlled with independent quartz crystal oscillators. After the deposition of the cathode, the substrates were transferred back into the glovebox. C60, the transport materials, AOB and BPhen were puried at least twice by vacuum gradient sublimation before use. DCV6T was purified once and DCV5T and the p-dopants were used as received. 
Each substrate contains four individual solar cells, the active area of which is definded by the overlap between ITO and Al. The solar cells had an average photovoltaic active area of $6-7 \mathrm{~mm}^{2}$. IV-characteristics of the solar cells were recorded in the glovebox using a Keithley Source Measure Unit (SMU236). Illumination was provided by a sun simulator SOL 1200 (Hoenle AG). Its intensity was controlled with a calibrated Si reference cell (Fraunhofer Institute for Solar Energy Systems, Freiburg). Intensity fluctuations of the sun simulator during IV-measurements were controlled using a photodiode.

The external quantum efficiency (EQE) was recorded using a home made setup. Using the EQE spectra of the solar cells the short circuit current density $J_{\mathrm{sc}}$ was corrected when calculating the device efficiency. Changes in open circuit voltage $V_{\text {oc }}$ and fill factor $F F$ due to the differences between real intensities and standard reporting conditions were not corrected.

To determine the absorption of the absorber materials pristine layers were evaporated on quartz glass substrates. The absorption of the thin film was subsequently measured in transmission with an UV-3100/MPC-3100 spectrometer (Shimadzu).

\subsection{Optical Simulations}

The used optical simulations are based on the transfer matrix algorithm. Refractive index $\mathrm{n}$ and absorption index $\mathrm{k}$ which serve as basis for the calculations were obtained from either fitting transmission and reflection data $^{16}$ or using ellipsometry. The calculations yield information on e.g. the distribution of optical field or photon absorption within the solar cell. With an absorber layer thickness of only a few ten nanometre the device stack is very sensitive to the stack design and the thicknesses of each layer needs to be chosen carefully. The simulations are used as basis for the stack design, especially for the development of stacked solar cell with a large number of layers.

\section{RESULTS AND DISCUSSION}

Based on the two oligothiophene derivates, solar cells were made and compared. The structure of the investigated devices with the thickness in nanometres and doping in weight percent given in brackets is:

$$
\begin{aligned}
& \text { A: ITO / Au (1) / p-4P-TPD (10, 19wt\%) / 4P-TPD (5) / DCV5T (12.3) / C60 (52) / BPhen (6) / Al (100) } \\
& \text { B: ITO / Au (1) / p-Di-NPB (20, 6wt\%) / Di-NPB (5) / DCV6T (10) / C60 (50) / BPhen (6) / Al (100) }
\end{aligned}
$$

The thin layer of Au on the ITO established a good ohmic contact between ITO and the HTM. Due to different values for the HOMO levels, 4P-TPD $(-5.6 \mathrm{eV})$ and Di-NPB $(-5.4 \mathrm{eV})$ were used as transport layer and p-doped with NDP-9 and NDP-2 respectively. An undoped interlayer of 4P-TPD and Di-NPB was inserted between transport and the absorber layer to reduce exciton quenching at dopant molecules. ${ }^{17}$ The photovoltaic active layer was realised as planar heterojunction at which the exciton separation takes place. The depostion of $\mathrm{Al}$ on BPhen is said to create defect states via which the electrons are transported. ${ }^{18}$

In figure 3 the absorption of the absorber materials as well as the EQE of both devices A and B is shown. The oligothiophene derivative have nearly the same absorption spectrum. With increasing chain length of the oligothiophenes, the absorption is is only slightly red-shifted. ${ }^{19}$ The absorption maximum in the case of DCV5T is at $577 \mathrm{~nm}$, the absorption maximum of DCV6T at $580 \mathrm{~nm}$. The second peak in their absorption spectrum at around 400nm is more pronounced in DCV5T than in DCV6T, where it is only a shoulder.

According to the EQE spectra the whole spectral region between $350 \mathrm{~nm}$ and $650 \mathrm{~nm}$ contributes to the current. In the spectral region between $350 \mathrm{~nm}$ and $500 \mathrm{~nm}$ mostly charge carriers produced in C60 contribute to the current density. This is attributed to the high thickness of the C60 layer which covers the optical interference maximum of this wavelength. At higher wavelength $(500-650 \mathrm{~nm})$ the current is produced by absorption in the oligothiophene. By comparison of the EQE spectra of both cells higher quantum efficiencies are observed for DCV5T than for DCV6T. This is not only caused by the thicker DCV5T layer in device A, but could also be affected by differences in material purity.

The corresponding current-voltage characterstics are shown in figure 4. Using the oligothiophene derivatives 

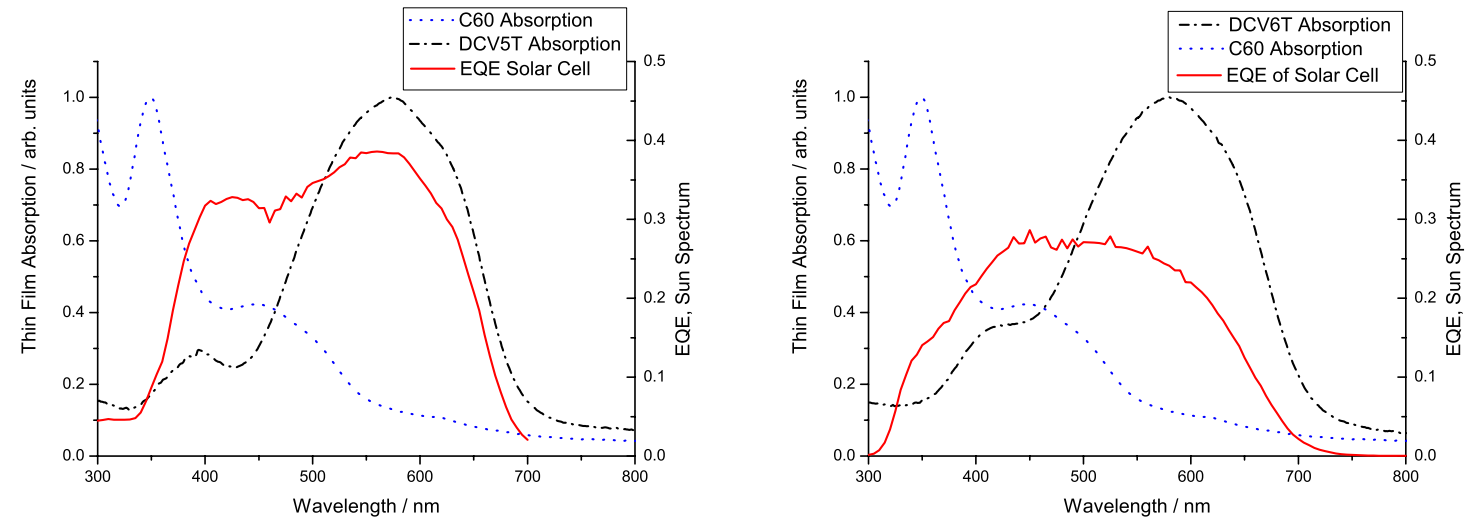

Figure 3. Absorption Spectra of the pristine absorber materials C60 (dotted line), DCV5T and DCV6T (both dashdotted lines) in thin films and EQE spectra (solid line) of device A (left) and B (right) with a planar heterojunction of DCV5T/C60 (left) and DCV6T/C60 (right) respectively.
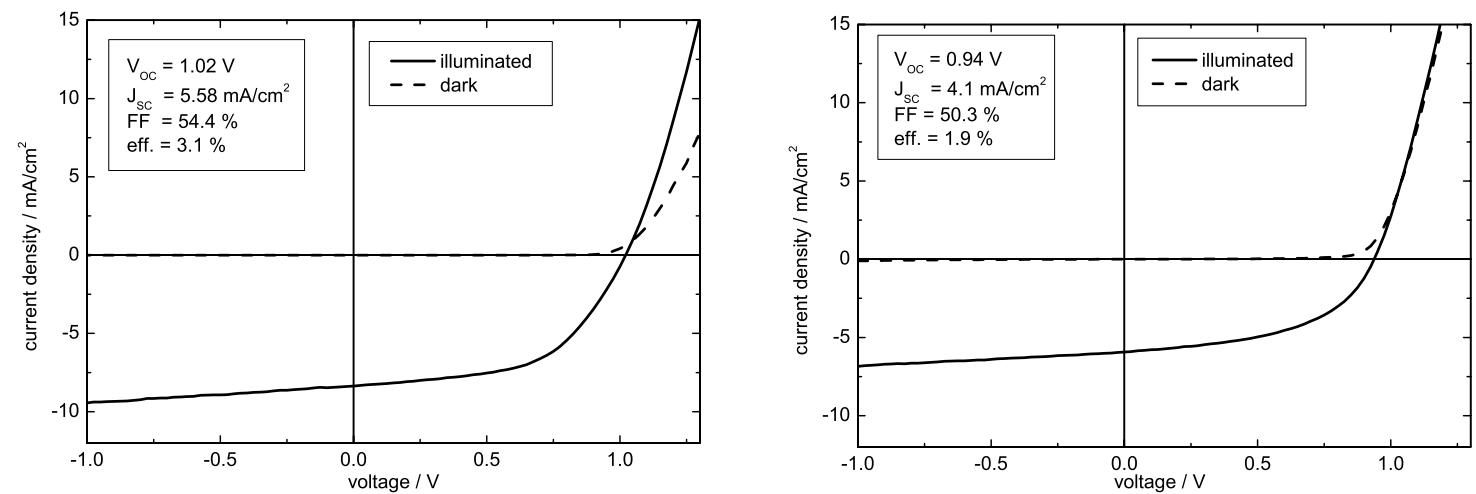

Figure 4. The current voltage characterstics of device A (a) and B (b) in the dark and under illumination. $J_{\text {sc }}$ given in the inset were corrected to the AM 1.5 sun spectrum by integrating the external quantum efficiency spectrum (Figure 3) with the AM1.5g spectrum. The calculation of the efficieny refers to the correct short circuit current density. 

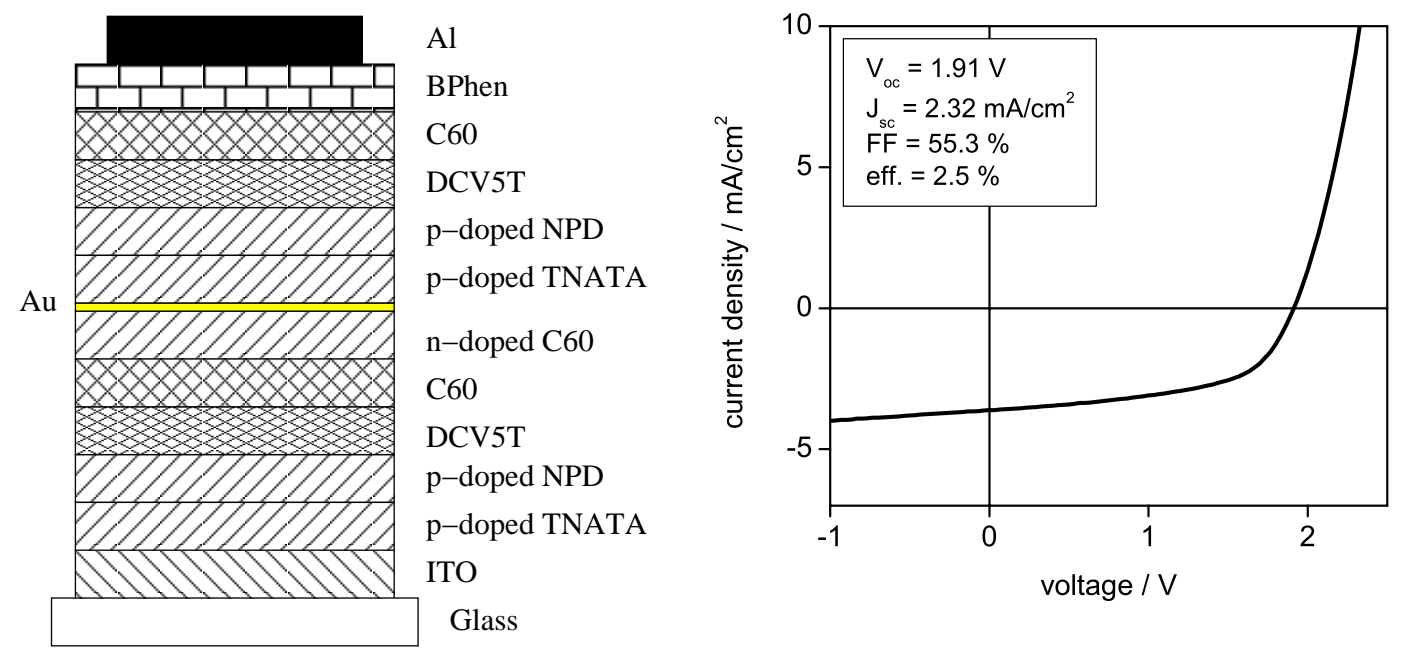

Figure 5. The structure (left) and the IV-characteristics (right) of the tandem solar cell C. It consists of of a p-i-n bottom and a p-i-i top cell, in which the absorber are realised as planar heterojunctions of DCV5T/C60. The current density was corrected using the same spectral mismatch according to the device A.

as photovoltaic electron donor, it is possible to reach in both cases very high $V_{\text {oc }}$ of $1 \mathrm{~V}$ and $0.9 \mathrm{~V}$ for DCV5T and DCV6T respectively. The difference is due to the different HOMOs of the oligothiophene in combination with $\mathrm{C} 60$ as electron acceptor. Using HTMs with higher HOMOs or reducing the doping ratio leads to energetic barriers in the charge extraction and an associated S-shape in the IV characteristics. ${ }^{11}$ In the presented solar cells the charge transport is sufficiently good as it is proven by the lack of the S-shape and the high $F F$.

\subsection{Tandem Solar Cells}

Tandem solar cells are realised by stacking two single solar cells on top of each other and connecting both in series through a recombination contact. ${ }^{12}$ In figure 5a such a tandem solar cell which comprises two planar heterojunctions of DCV5T/C60 is shown. The stack structure has been chosen such that the two solar cells are positioned in two different maxima of the optical interference pattern. The layer sequence with the layer thickness given in nanometres and doping in weight percent given in brackets is:

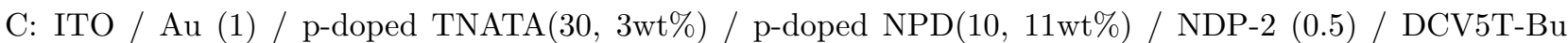
(12.3) / C60 (52) / n-doped C60 (13, 1.4wt\%) / Au(1) / p-doped TNATA(130, 3\%) / p-doped NPD(10, $11 \mathrm{wt} \%) /$ NDP-2(0.5) / DCV5T-Bu (8.6) / C60 (52)/ BPhen (6) / Al (100)

The bottom solar cell is realised in p-i-n structure using n-doped C60 as electron transport material, the top solar cell again in p-i-i structure. The recombination contact is established through the incorporation of $\mathrm{Au}$ clusters between the n-doped C60 and p-doped TNATA. NDP-2 was used as dopant here.

Even though both solar cells cover the same spectral region, good results have been obtained. An open circuit voltage of $1.91 \mathrm{~V}$, a current density of approx. $2.32 \mathrm{~mA} / \mathrm{cm}^{2}$ and a fill factor of $55.3 \%$, leading to a power efficiency of approx. 2.5\%. A saturation factor as the relation between the current density at $-1 \mathrm{~V}$ to $J_{\mathrm{sc}}$ is observed to be 1.1. The good $F F$ and the low saturation factor show that the recombination contact is working efficiently. However, the current of the tandem device is less then in the single solar cell due to the differences in positions in the optical interference pattern and to the same spectral absorption region of the two single solar cells. The smallest current limits the solar cell and current matching is not sufficient. The efficiencies was estimated using the mismatch from the single solar cell, being aware of the fact that this is only a very crude assumption. However, so far we have not been able to reliably measure the EQE of organic tandem solar cells. 
The presented tandem device is a proof of principle to see whether it is possible to stack solar cells with very high open circuit voltages and still obtain nearly the sum of $V_{\text {oc }}$ of both solar cells. The shown efficient recombination contact is the key to efficiently stack solar cells. Given that, the next main step is to use different absorber materials with different spectral sensitivities in the single solar cells. Further progress is expected when materials absorbing in complimentary spectral regions will be used.

\section{CONCLUSION}

We have shown results for solar cells using oligothiophene derivatives in combination with C60 as photovoltaic active heterojunction. Devices made with this material combination reach very high open circuit voltage of above $0.9 \mathrm{~V}$ and fill factors above $50 \%$. Their efficiency is up to approx. $3.1 \%$ and is currently limited by their photocurrent in the order of $5-6 \mathrm{~mA} / \mathrm{cm}^{2}$. The DCVnTs are one of the first new small molecule absorbers with efficiencies comparable or even exceeding the ones of the reference material combination of metal-phthalocyanines and $\mathrm{C} 60$.

One of the big advantages of evaporated solar cells is the simple realisation of many stacked layers, e.g. for tandem solar cells. Such a device has been realised with two stacked solar cells, each with a planar heterojunction of DCV5T/C60. Through a very efficient recombination zone between in a tandem device the resulting $V_{\text {oc }}$ nearly reaches the sum of the $V_{\mathrm{oc}}$ of the two serially connected solar cells, i.e. $1.9 \mathrm{~V}$. Further optimisation of the stack design to maximise the current of the complete cell will further improve the solar cells.

\section{ACKNOWLEDGMENTS}

We thank Selina Oltholf for the UPS measurements. This work is supported as InnoProfile project 03IP602 by the German Ministry for Research and Education (BMBF) and by the Sächsische Aufbaubank - Förderbank $(\mathrm{SAB})$.

\section{REFERENCES}

[1] Green, M. A., Emery, K., King, D. L., Hisikawa, Y., and Warta, W., "Solar cell efficiency tables (version 29)," Progress in Photovoltaics 15(1), 35-40 (2007).

[2] Lungenschmied, C., Dennler, G., Neugebauer, H., Sariciftci, S. N., Glatthaar, M., Meyer, T., and Meyer, A., "Flexible, long-lived, large-area, organic solar cells," Solar Energy Materials and Solar Cells 91, 379-384 (2007).

[3] G24i. http://www.g24i.com/. [Online; accessed 20-Jan-2007].

[4] Konarka. http://www.konarka.com. [Online; accessed 20-Jan-2007].

[5] Heliatek GmbH. http://www.heliatek.com. [Online; accessed 20-Jan-2007].

[6] Plextronics. http://www.plextronics.com/. [Online; accessed 20-Jan-2007].

[7] Li, B., Wang, L., Kang, B., Wang, P., and Qiu, Y., "Review of recent progress in solid-state dye-sensitized solar cells," Solar Energy Materials and Solar Cells 90, 549-573 (2006).

[8] Günes, S., Neugebauer, H., and Sariciftci, N. S., "Conjugated polymer-based organic solar cells," Chemical Reviews 107, 1324-1338 (2007).

[9] Rand, B. P., Genoe, J., Heremans, P., and Poortmans, J., "Solar cells utilizing small molecular weight organic semiconductors," Progress in Photovoltaics 15, 659-676 (2007).

[10] Schueppel, R., Schmidt, K., Uhrich, C., Schulze, K., Wynands, D., Brédas, J. L., Brier, E., Reinold, E., Bu, H.-B., Baeuerle, P., Maennig, B., Pfeiffer, M., and Leo, K., "Optimizing organic photovoltaics using tailored heterojunctions: A photoinduced absorption study of oligothiophenes with low band gaps," Physical Review $B$ 77, 085311 (2008).

[11] Schulze, K., Uhrich, C., Schppel, R., Leo, K., Pfeiffer, M., Brier, E., Reinold, E., and Baeuerle, P., "Efficient vacuum-deposited organic solar cells based on a new low-bandgap oligothiophene and fullerene C60," Advanced Materials 18(21), 2872-2875 (2006). 
[12] Maennig, B., Drechsel, J., Gebeyehu, D., Simon, P., Kozlowski, F., Werner, A., Li, F., Grundmann, S., Sonntag, S., Koch, M., Leo, K., Pfeiffer, M., Hoppe, H., Meissner, D., Sariciftci, N. S., Riedel, I., Dyakonov, V., and Parisi, J., "Organic p-i-n solar cells," Applied Physics A-Materials Science E Processing 79(1), 1-14 (2004).

[13] Drechsel, J., Maennig, B., Gebeyehu, D., Pfeiffer, M., Leo, K., and Hoppe, H., "MIP - type organic solar cells incorporating phthalocyanine/fullerene mixed layers and doped wide-gap transport layers," Organic Electronics 5, 175-186 (2004).

[14] Persson, N. and Inganas, O., "Organic tandem solar cells - modelling and predictions," Solar Energy Materials and Solar Cells 90, 3491-3507 (2006).

[15] Mitsumoto, R., Araki, T., Ito, E., Ouchi, Y., Seki, K., Kikuchi, K., Achiba, Y., Kurosaki, H., Sonoda, T., Kobayashi, H., Boltalina, O. V., Pavlovich, V. K., Sidorov, L. N., Hattori, Y., Liu, N., Yajima, S., Kawasaki, S., Okino, F., and Touhara, H., "Electronic structures and chemical bonding of fluorinated fullerenes studied by NEXAFS, UPS, and vacuum-UV absorption spectroscopies," Journal of Physical Chemistry A 102, 552560 (1998).

[16] Fritz, T., Hahn, J., and Bottcher, H., "Determination of the optical constants of evaporated layers," Thin Solid Films 2, 249-257 (1989).

[17] Schulze, K., Uhrich, C., Schueppel, R., Leo, K., Pfeiffer, M., Brier, E., Reinold, E., and Baeuerle, P., "Efficient heterojunction organic solar cells with high photovoltage containing a low gap oligothiophene derivative," Proceedings of SPIE: Organic Optoelectronics and Photonics II 6192, 61920C, SPIE (2006).

[18] Peumans, P. and Forrest, S., "Very-high-efficiency double-heterostructure copper phthalocyanine/C-60 photovoltaic cells," Applied Physics Letters 97, 126-128 (2001).

[19] Schueppel, R., Schmidt, K., Uhrich, C., Schulze, K., Wynands, D., Brédas, J. L., Maennig, B., Pfeiffer, M., Leo, K., Brier, E., Reinold, E., Bu, H.-B., and Baeuerle, P., "Tailored heterojunctions for efficient thin-film organic solar cells: a photoinduced absorption study," Proceedings of SPIE: Organic Photovoltaics VIII 6656, 66560G, SPIE (2006). 